Research Article

\title{
Drillstring Oscillations: The Influence of Fluid Loading and Stabilizer Effects
}

\author{
Yongsheng Liu $\mathbb{D},{ }^{1,2}$ Deli Gao, ${ }^{3}$ Vipin Agarwal, ${ }^{4}$ Xie Zheng, ${ }^{5}$ and Balakumar Balachandran ${ }^{5}$ \\ ${ }^{1}$ School of Engineering and Technology, China University of Geosciences, Beijing 10083, China \\ ${ }^{2}$ Key Laboratory of Deep Geological Drilling Technology of Ministry of Natural Resources, Beijing, China \\ ${ }^{3}$ MOE Key Lab of Petroleum Engineering, China University of Petroleum-Beijing, Beijing 102249, China \\ ${ }^{4}$ Department of Mechanical Engineering, University of Michigan, Ann Arbor, MI 48109, USA \\ ${ }^{5}$ Department of Mechanical Engineering, University of Maryland, College Park, MD 20742, USA \\ Correspondence should be addressed to Yongsheng Liu; yongsheng@cugb.edu.cn
}

Received 1 June 2020; Revised 10 August 2020; Accepted 17 May 2021; Published 25 May 2021

Academic Editor: Jinqiang Gan

Copyright ( $) 2021$ Yongsheng Liu et al. This is an open access article distributed under the Creative Commons Attribution License, which permits unrestricted use, distribution, and reproduction in any medium, provided the original work is properly cited.

Drillstring vibrations can be undesirable for drilling operations. Here, attention is focused on vibrations of the upper portion of a drillstring as these vibrations can cause drillpipe wear and casing wear. A reduced-order model is developed to study the motions of a drillstring by taking fluid loading and stabilizer effects into account. In this model development, the distributed nature of the fluid loading is taken into account, and the drillstring is treated as a beam structure. Perturbation analyses are carried out with the reduced-order system, and the system responses are examined for primary and secondary (subharmonic and superharmonic) resonance excitations. The analytical-numerical results reveal the rich nature of the system behavior and help understand the drillstring motions during various resonance conditions.

\section{Introduction}

Deep petroleum exploration and mining operations have become unavoidable for tapping into unconventional oil and gas resources [1]. Drilling is the commonly used mode for mining of these resources. In Figure 1, a schematic of a rotary drilling process is illustrated. The drillstring is a principal component of the drilling equipment. This component is used for rock breaking to form a borehole as well as to convey the drill mud to the bottom of the drill well. While in operation, a drillstring is subjected to fluctuating fluid forces from the environment it is immersed in and constrained motions within a wellbore. A drillstring can experience different vibration and deformation states under different working conditions, including transverse vibrations, torsional vibrations, longitudinal vibrations, and coupled forms of these vibrations. Due to the complex force conditions and constraint of the wellbore, the deformation of a drillstring is also varied, with the possibilities including bending, sinusoidal distortion [3], and spiral buckling [4].
Usually, the configuration of the drillstring is a combination of various deformation states. These irregular deformations and vibrations can lead to the failure of the drillstring itself $[5,6]$ and also cause wear of downhole tools and casing [7-9] in contact with the drillstring. An understanding of downhole vibrations and deformations of a drillstring is an important basis for guaranteeing drilling safety for complex structural wells and for providing design support for optimized drilling.

While vibrations of drillstrings can be beneficial for enhancing drilling efficiency, depending on the vibration level, they can also lead to the failure of a drillstring. Many researchers have established various reduced-order models and carried out numerical studies with them, in an attempt to better understand an actual drilling operation. Apostal et al. [10] examined the lateral vibrations of a drillstring by employing the finite element method. They also considered damping during failure analysis based on frequency responses. Inspired by rotor dynamics, Jansen [11] modeled the drillstring model with a rotor dynamics model and 


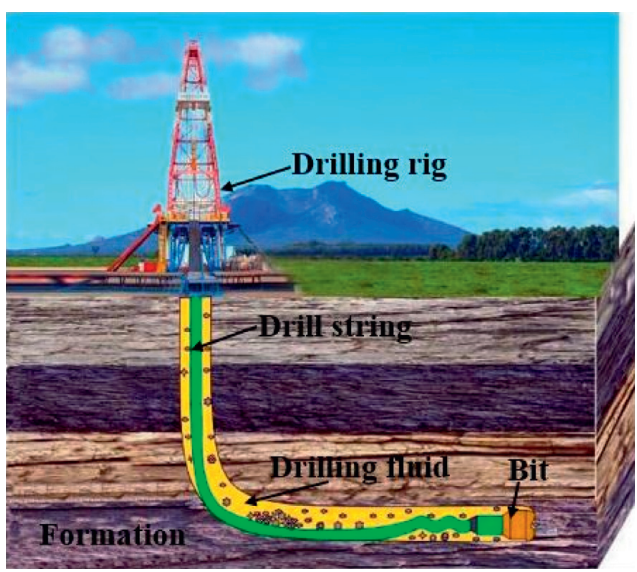

FIGURE 1: Schematic of rotary drilling from authors' prior work [2].

considered the effects of drilling fluid, stabilizer clearance, and stabilizer friction. Spanos and Payne [12] also used the finite element technique to model the bottom hole assembly (BHA) dynamics and took the fluid added-mass effect into consideration. They modeled the BHA excitation as a monochromatic function of time and obtained noteworthy results. Vaz and Patel [13] carried out an investigation into drillstring dynamic stability and static deflection. Transverse vibrations were considered in this work. Yigit and Christoforou [14] studied a dynamic model, wherein the axial and transverse vibrations were coupled. Their results revealed the importance of considering the coupled behavior, in particular, for determining stability. Following prior studies, Khulief and Al-Naser [15] conducted finite element analysis to include the BHA and drillpipe sections. Sahebkar et al. [16] examined natural frequencies and mode shapes of a drillstring system in an inclined well. They investigated the effects of axial compression load, imbalance mass, and nonlinear fluid force on the drillstring response. In recent work, Liu et al. [17, 18] examined multiple regenerative effects and state-dependent delay effects in the context of drillstring dynamics. These studies revealed the complex nature of drillstring dynamics, in particular, stick-slip and whirling dynamics.

A considerable amount of work has been reported in the literature on drillstring dynamics, in particular, in the BHA region. By contrast, the dynamics of the upper portion of the drillstring system, which is important for casing wear, has received limited attention. As the upper portion is removed from the region of cutting action, the influence of the cutting process may be neglected in modeling the vibrations of the upper portion [19-21]. Furthermore, given the challenges encountered with placement of downhole tools $[2,22]$, the forces generated by the drilling fluid are important to consider. However, the fluid effects on drillstring vibrations have not received complete consideration. In this work, the authors mainly focus on constructing analytical approximations for better understanding the nonlinear behavior of drillstrings subjected to fluid forcing. Compared to previous studies, here, the drillstring with the stabilizer is modeled as a beam with a concentrated mass, and the stabilizer force is captured through a nonlinear model. This is the first time that such a nonlinear constraint associated with a stabilizer has been considered. The distributed parameter system governing the drillstring system is reduced to an ordinary differential equation by using a Galerkin procedure, and the behavior is examined by using weakly nonlinear analyses for different external resonance conditions. Response curves are generated and discussed with regard to the stability of solutions and sensitivity to parameters.

\section{Model Development}

2.1. Simplified Model. As shown in Figure 1, the drillstring is a flexible body, whose length can be several kilometers. Stabilizers are located at certain distances along with the drillstring to enhance lateral stability. For the convenience of research, a representative part between two stabilizers is taken as the region of interest, as shown on the left side of Figure 2. With this representation, one can simplify the system as a beam structure with a discrete mass element, as shown on the right side of Figure 2, wherein the stabilizer $(M)$ is located at a distance $h$ from the upper end. Due to the uncertain interaction between the stabilizer and the borehole, a nonlinear force $F_{s}$ is acted on the discrete mass element. Following prior studies [23], the form of this nonlinear force can be expressed as $F_{s}=k_{1} y+k_{2} y^{3}$, where $k_{1}$ is the linear spring stiffness and $k_{2}$ is the cubic nonlinear spring stiffness.

The influence of drill mud is acting throughout the length of the beam. The force form can be simplified as a harmonic distributed force with an amplitude of $P(\mathrm{~N} / \mathrm{m})$. The damping force associated with the fluid around the drillstring is also considered. This force has the form $F_{f}=(1 / 2) C_{f}(\partial y / \partial t)$, where $C_{f}$ is the viscous damping coefficient (N.s $\left./ \mathrm{m}^{2}\right)$ [24]. The length of the beam section is assumed to be $l$, and mass per unit length is $m$. Considering the top end connection to the upper drillpipe, a rotational joint spring with stiffness $K_{r}$ is included at this end.

The lower end of the section is free or connected to the bottom hole assembly (BHA) or other downhole tools; a spring force with stiffness $K_{t}$ is used in the modeling.

2.2. Governing Equations. Making use of Bernoulli-Euler beam theory and the depiction shown in Figure 2, the governing equation of motion in the open domain $0<y<l$ can be written as [25]

$$
E I \frac{\partial^{4} y}{\partial x^{4}}+m \frac{\partial^{2} y}{\partial t^{2}}=-\left(k_{1} y+k_{2} y^{3}\right) \delta(x-a)+P \cos \left(\omega_{f} t\right)-M \frac{\partial^{2} y}{\partial t^{2}} \delta(x-h)-\frac{1}{2} C_{f} \frac{\partial y}{\partial t} .
$$




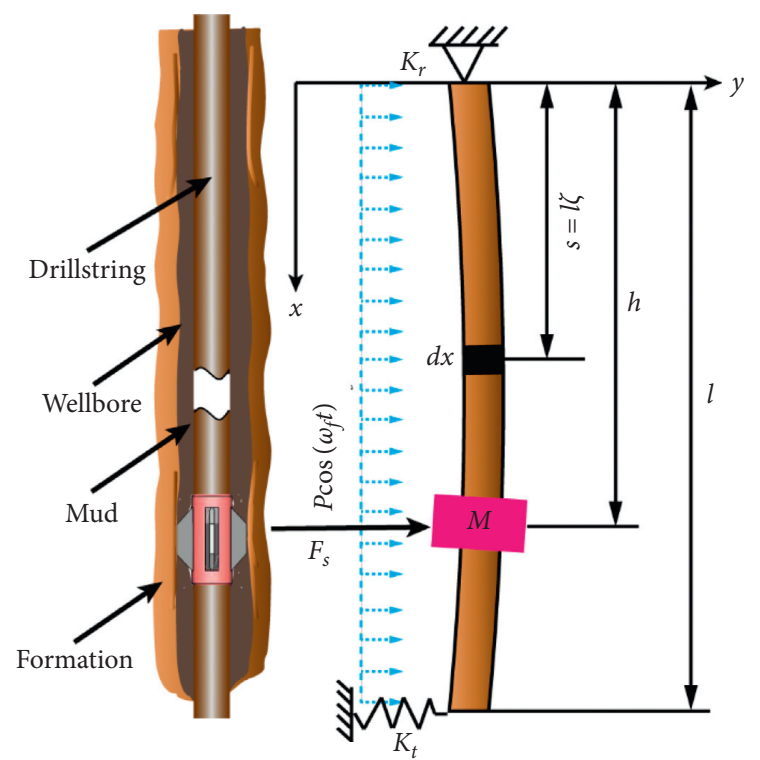

FIGURE 2: Simplified model to account for fluid forces and stabilizer effects.

Here, $y(x, t)$ is the transverse vibration displacement, and $m$ is the mass per unit length $(\mathrm{kg} / \mathrm{m})$. For convenience, the nondimensional parameters for span lengths $\xi=x / l$ and $\eta=h / l$, the mass ratio $\mu=M / m$, and the amplitude $w=y / l$ are introduced. In addition, the other related nondimensional variables are used $\left(\tau^{\prime}=t / l^{2} \cdot \sqrt{E I / m}, K_{1}=k_{1} l^{3} / E I\right.$, $K_{2}=k_{2} l^{5} / E I, \quad P^{\prime}=P l^{3} / E I, \quad C_{f}^{\prime}=C_{f} l^{2} / \sqrt{E I / m}$, and $\left.\omega=\omega_{f} l^{2} / \sqrt{E I / m} \cdot\right)$. Thus, the governing equation for the system in the open domain can be converted into the following form:

$$
\begin{aligned}
w^{\prime \prime \prime}+[1+\mu \delta(\xi-\eta)] \ddot{w}= & -\left(K_{1} w+K_{2} w^{3}\right) \delta(\xi-\eta) \\
& +P^{\prime} \cos \left(\omega \tau^{\prime}\right)-\frac{1}{2} C_{f}^{\prime} \dot{w} .
\end{aligned}
$$

Here, the overdot denotes the derivative with respect to the dimensionless time $\tau^{\prime}$, while the prime $\left({ }^{\prime}\right)$ represents the derivative with respect to the dimensionless spatial variable $\xi$. The boundary conditions can be obtained as

$$
\begin{aligned}
w\left(0, \tau^{\prime}\right) & =\frac{\partial^{2} y\left(1, \tau^{\prime}\right)}{\partial \xi^{2}}=0, \\
K_{r} \frac{\partial w\left(0, \tau^{\prime}\right)}{\partial \xi} & =E I \frac{\partial^{2} w\left(0, \tau^{\prime}\right)}{\partial \xi^{2}}, \\
K_{t} w\left(1, \tau^{\prime}\right) & =E I \frac{\partial^{3} w\left(1, \tau^{\prime}\right)}{\partial \xi^{3}} .
\end{aligned}
$$

2.3. Discretization, Linear System, and Nonlinear System. The Galerkin technique is used to go simplify the governing partial differential equation to a set of ordinary differential equations. To this end, the solution is assumed to be in the form

$$
w(\xi, \tau)=\phi(\xi) q\left(\tau^{\prime}\right),
$$

where $\phi(\xi)$ is the mode shape function, which depends on the geometric and boundary conditions of the beam. $q\left(\tau^{\prime}\right)$ is the corresponding generalized coordinate. Substituting (4) into (2), multiplying by $\phi(\xi)$, and integrating from 0 to 1 , one obtains

$$
c_{1} \ddot{q}+c_{f} \dot{q}+c_{2} q+c_{3} q^{3}=P_{0} \cos \left(\omega \tau^{\prime}\right)
$$

whereas all the coefficients of the above equation are relating to the mode shape function. The concrete expression of each coefficient is listed as follows:

$$
\begin{aligned}
& c_{1}=\int_{0}^{1} \phi(\xi)^{2} \mathrm{~d} \xi+\mu \phi(\eta)^{2} \\
& c_{2}=\int_{0}^{1} \phi(\xi) \phi(\xi)^{\prime \prime \prime} \mathrm{d} \xi+K_{1} \int_{0}^{1} \phi(\eta)^{2} \mathrm{~d} \xi \\
& c_{3}=K_{2} \int_{0}^{1} \phi(\eta)^{4} \mathrm{~d} \xi \\
& c_{f}=\frac{C_{f}^{\prime}}{2} \int_{0}^{1} \phi(\xi)^{2} \mathrm{~d} \xi \\
& P_{0}=P^{\prime} \int_{0}^{1} \phi(\xi) \mathrm{d} \xi .
\end{aligned}
$$

For convenience, equation (5) is converted into the following form:

$$
\ddot{q}+q+\alpha_{1} \dot{q}+\alpha_{2} q^{3}=f_{0} \cos (\Omega \tau)
$$

where

$$
\begin{aligned}
\alpha_{1} & =\frac{c_{f}}{c_{2}} \sqrt{\frac{c_{1}}{c_{2}}}, \\
\alpha_{2} & =\frac{c_{3}}{c_{2}} \\
f_{0} & =\frac{P_{0}}{c_{2}}, \\
\tau & =\sqrt{\frac{c_{2}}{c_{1}}} \tau^{\prime},
\end{aligned}
$$

and $\Omega=\omega / \omega_{0}$ is the nondimensional excitation frequency.

In fact, the eigenfunctions, also called mode shape functions, of any vibration beam can be inherited from the base beam mode [25]. Combining the boundary conditions, which could introduce the springs $K_{r}$ and $K_{t}$, this basic equation can obtain the eigenfunctions which can capture the shape characteristic of the base beam. The transcendental frequency of this problem is given by reference.

$$
\left(\frac{S_{1}}{p}\right)(1+\cos p \cos h p)+\left[\frac{2 S_{2}}{p^{3}}\right] \sin p \sin h p+\cos p \sin h p
$$

$-\sin p \cos h p+\left[\frac{S_{1} S_{2}}{p^{4}}\right](\sin p \cos h p-\cos p \sin h p)=0$. 
Finally, the eigenfunctions are defined as

$$
\phi(\xi)=\left(\frac{1}{\varsigma}\right)[\sin p \xi-A \sin h p \xi-B(\cos p \xi-\cos h p \xi)]
$$

where $S_{1}=K_{r} l / E I$ and $S_{2}=K_{t} l^{3} / E I$ are dimensionless stiffness parameters, $\varsigma=\phi(1)$ is used to realize normalization, $p^{4}=m \omega_{0}^{2} l^{4} / E I$ denotes the dimensionless frequency parameter ( $\omega_{0}$ is the natural frequency of the base beam), and $A$ and $B$ are weighting constants associated with each mode; the expressions are

$$
\begin{aligned}
& A=\frac{(S / 2 p)(\cos p+\cos h p)-\sin p}{(S / 2 p)(\cos p+\cos h p)+\sinh p} \\
& B=\frac{\sin p+A \sin h p}{\cos p+\cos h p} .
\end{aligned}
$$

It is obvious to figure out that the boundary situations of the beam are determined by parameters $S_{1}$ and $S_{2}$ such as the combination parameters $\left(S_{1} \longrightarrow 0, S_{2} \longrightarrow 0\right),\left(S_{1} \longrightarrow \infty\right.$, $\left.S_{2} \longrightarrow 0\right),\left(S_{1} \longrightarrow 0, S_{2} \longrightarrow \infty\right)$, and $\left(S_{1} \longrightarrow \infty, S_{2} \longrightarrow \infty\right)$ represent the case of hinged-free, clamped-free, simply supported, and clamped-simply supported beams, respectively. According to different boundary parameters, one can obtain the eigenfrequency from equation (10), in general, which has an infinite number of roots $p_{i}$. And for each $p_{i}$, there will be a natural frequency and a mode shape corresponding to it. For the clamped-free case, here, it is assumed that $S_{1}=10^{7}$ and $S_{2}=0$, and the first four frequencies will be $1.8751,4.6941,7.8547$, and 10.9955 [25].

\section{Perturbation Solution for the Response}

For the governing equation with the cubic nonlinearity term and small excitation amplitude, the standard multiple-scales method is used to obtain the approximate solution. Based on this method, the solution for the response can be written as

$$
q(\tau, \varepsilon)=q_{0}\left(T_{0}, T_{1}\right)+\varepsilon q_{1}\left(T_{0}, T_{1}\right)+\cdots,
$$

wherein

$$
\begin{aligned}
& T_{0}=\tau, \\
& T_{1}=\varepsilon \tau, \\
& \cdots \\
& T_{n}=\varepsilon^{n} \tau,
\end{aligned}
$$

and the derivative operator $\left(D_{n}=d / d T_{n}\right)$ is introduced which results in different derivatives as follows:

$$
\begin{aligned}
\frac{\mathrm{d}}{\mathrm{d} \tau} & =D_{0}+\varepsilon D_{1}+\varepsilon^{2} D_{2}+\cdots \\
\frac{\mathrm{d}^{2}}{\mathrm{~d} \tau^{2}} & =D_{0}^{2}+2 D_{0} D_{1} \varepsilon+\left(D_{1}^{2}+2 D_{0} D_{2}\right) \varepsilon^{2}+\cdots
\end{aligned}
$$

3.1. Primary Resonance. Considering weak damping and the small excitation term, equation (7) can alternatively be written as

$$
\ddot{q}+q=\varepsilon\left[-\alpha_{1} \dot{q}-\alpha_{2} q^{3}+f_{0} \cos (\Omega \tau)\right] .
$$

Primary resonance means the excitation frequency close to the natural frequency. Here, one assumes the nondimensional excitation frequency is expressed as

$$
\Omega=1+\varepsilon \sigma,
$$

where $\sigma$ is the detuning parameter. After substituting equations (13) and (16) into (15) and extracting the coefficients of each order of $\varepsilon$ from zero to 1 , it yields

$$
\begin{gathered}
\varepsilon^{0}: D_{0}^{2} q_{0}+q_{0}=0 \\
\mathcal{\varepsilon}^{1}: D_{0}^{2} q_{1}+q_{1}= \\
+\frac{2 D_{0} D_{1} q_{0}-\alpha_{1} D_{1} q_{0}-\alpha_{2} q_{0}^{3}}{2 f_{0}\left(e^{i\left(\sigma T_{1}+T_{0}\right)}+\text { c.c }\right)}
\end{gathered}
$$

where c.c indicates the complex conjugate. The solution of the second-order ordinary differential equation (17) can be expressed as

$$
q_{0}=a\left(T_{1}\right) \cos \left[T_{0}+B\left(T_{1}\right)\right]
$$

in which $a$ and $B$ are real functions of $T_{1}$. Substituting equation (19) into equation (18) and doing some simplification yield in

$$
\begin{aligned}
D_{0}^{2} q_{1}+q_{1} & =-\frac{\alpha_{2} a^{3} e^{3 i T_{1}} e^{3 i B}}{8}-\frac{3 \alpha_{2} a^{3} e^{i T_{1}} e^{i B}}{8}-\frac{i \alpha_{1} a e^{i T_{1}} e^{i B}}{2}+\frac{i \alpha_{1} a e^{-i T_{1}} e^{-i B}}{2} \\
& -i a^{\prime} e^{i T_{1}} e^{i B}+a B^{\prime} e^{i T_{1}} e^{i B}+\frac{f_{0}}{2} e^{i \sigma T_{1}} e^{i T_{1}}+\text { c.c }
\end{aligned}
$$

where the sign of prime denotes the derivative with respect to $T_{1}$. Now, one can separate the secular term from equation
(20), and let the sum of the secular term's coefficient be equal to zero. 


$$
\frac{3 \alpha_{2} a^{3} e^{i T_{1}} e^{i B}}{8}+\frac{i \alpha_{1} a e^{i B}}{2}+i a^{\prime} e^{i T_{1}} e^{i B}-a B^{\prime} e^{i B}-\frac{f_{0}}{2} e^{i \sigma T_{1}}=0 .
$$

Multiplying $e^{i B}$ to equation (21) and introducing $C=\sigma T_{1}-B$, with rearrangement, it results in

$$
\frac{3 \alpha_{2} a^{3}}{8}+\frac{i \alpha_{1} a}{2}+i a^{\prime}-a\left(\sigma-C^{\prime}\right)-\frac{e^{i C}}{2}=0 .
$$

Converting equation (22) into a trigonometric form and separating the result into real and imaginary parts, one can obtain the modulation equations:

$$
\begin{aligned}
& \text { real part: } \frac{3 \alpha_{2} a^{3}}{8}-a \sigma+a \frac{\mathrm{d} C}{\mathrm{~d} T_{1}}-\frac{f_{0} \cos (C)}{2}=0, \\
& \text { imaginary part: } \frac{\alpha_{1} a}{2}+\frac{\mathrm{d} a}{\mathrm{~d} T_{1}}-\frac{f_{0} \sin (C)}{2}=0 .
\end{aligned}
$$

When $\mathrm{d} C / \mathrm{d} T_{1}$ and $d a / d T_{1}$ toward to zero, equation (23) reflects the steady-state response of the system.
3.2. Secondary Resonances. This section will focus on finding the secondary resonances that can occur in the system. Firstly, equation (7) will be converted into the following form:

$$
\ddot{q}+q=\varepsilon\left[-\alpha_{1} \dot{q}-\alpha_{2} q^{3}\right]+f_{0} \cos (\Omega \tau) .
$$

Substituting equation (12) into equation (24) and collecting the coefficient terms of $\varepsilon$, it shows that

$$
\begin{gathered}
\mathcal{\varepsilon}^{0}: D_{0}^{2} q_{0}+q_{0}=f_{0} \cos (\Omega \tau), \\
\varepsilon^{1}: D_{0}^{2} q_{1}+q_{1}=-2 D_{0} D_{1} q_{0}-\alpha_{1} D_{1} q_{0}-\alpha_{2} q_{0}^{3} .
\end{gathered}
$$

The solutions of equation (25) can be written as

$$
q_{0}=A\left(T_{1}\right) e^{i T_{0}}+\Lambda \times e^{i \Omega T_{0}}+\text { c.c },
$$

where $A\left(T_{1}\right)$ contains the amplitude and phase information and $\Lambda$ is equal to $f_{0} / 2\left(1-\Omega^{2}\right)$. When putting equation (27) into equation (26), it results in

$$
\begin{aligned}
D_{0}^{2} q_{1}+q_{1}= & -6 \alpha_{2} A \Lambda^{2} e^{i T_{0}}-i \alpha_{1} A e^{i T_{0}}-2 i A^{\prime} e^{i T_{0}}-3 \alpha_{2} A^{2} \bar{A} e^{i T_{0}}-\alpha_{2} A^{3} \Lambda e^{3 i T_{0}} \\
& -\alpha_{2} \Lambda^{3} e^{3 i \Omega T_{0}}-3 \alpha_{2} A^{2} \Lambda e^{i(\Omega+2) T_{0}}-3 \alpha_{2} \bar{A}^{2} \Lambda e^{i(\Omega-2) T_{0}} \\
& -3 \alpha_{2} A \Lambda^{2} e^{i(2 \Omega+1) T_{0}}-3 \alpha_{2} \bar{A} \Lambda^{2} e^{i(2 \Omega-1) T_{0}}-i \alpha_{1} \Lambda \Omega e^{i \Omega T_{0}} \\
& -6 \alpha_{2} A \bar{A} \Lambda e^{i \Omega T_{0}}-3 \alpha_{2} \Lambda^{3} e^{i \Omega T_{0}}+\text { c.c, }
\end{aligned}
$$

where $\bar{A}$ is the conjugate of $A$. Equation (28) can be used to determine the form of secondary resonance based on different $\Omega$, which determines whether the secular term appears or not. Judging from the right-hand side of equation (28), one can obtain that the system will show 1/3-order subharmonic and 3rd-order superharmonic resonances. The detailed description of subharmonic and superharmonic resonances is as follows.

3.2.1. Subharmonic Resonance. Based on the aforementioned analysis and introducing the detuning parameter $\sigma$, the dimensionless excitation frequency $\Omega$ could be expressed as

$$
\Omega=3+\varepsilon \sigma .
$$

Substituting equation (29) into equation (28) and extracting the secular term,

$$
3 e^{i T_{1}} \alpha_{2} \Lambda \bar{A}^{2}+3 \alpha_{2} A^{2} \bar{A}+6 \alpha_{2} A \Lambda^{2}+i A \alpha_{1}+2 i A^{\prime}=0 .
$$

The complex amplitude $A$ can be expressed as

$$
A=\frac{1}{2} a\left(T_{1}\right) e^{i \beta\left(T_{1}\right)},
$$

where $a$ and $\beta$ are the amplitude and phase, respectively. Substituting equation (31) into equation (30) and introducing $C=\sigma T_{1}-3 \beta$ to simplify the result, it yields

$$
\frac{3 \alpha_{2} \Lambda a^{2} e^{i C}}{4}+\frac{3 \alpha_{2} a^{3}}{8}-a\left(\frac{1}{3} \sigma-\frac{1}{3} C^{\prime}\right)+3 \alpha_{2} a \Lambda^{2}+\frac{i \alpha_{1} a}{2}+i a^{\prime}=0 .
$$

From equation (32), separating real and imaginary parts, one can obtain the modulation equations as follows:

$$
\begin{aligned}
a^{\prime} & =-\frac{\alpha_{1} a}{2}-\frac{3 \alpha_{2} \Lambda a^{2} \sin (C)}{4}, \\
a C^{\prime} & =-\frac{9 \alpha_{2} \Lambda a^{2} \cos (C)}{4}-\frac{9 \alpha_{2} a^{3}}{8}+a \sigma-9 a \alpha_{2} \Lambda^{2} .
\end{aligned}
$$

For the steady-state response of the system, the derivative terms $a^{\prime}$ and $C^{\prime}$ will approach to zero.

3.2.2. Superharmonic Resonance. As for the third-order superharmonic resonance, the dimensionless excitation frequency $\Omega$ can be written as

$$
3 \Omega=1+\varepsilon \sigma .
$$

Like the former process, using equations (28) and (34), the secular term is derived as

$$
e^{3 i \sigma T_{1}} \alpha_{2} \Lambda^{3}+3 \alpha_{2} \bar{A} A^{2}+6 \alpha_{2} A \Lambda^{2}+i A \alpha_{1}+2 i A^{\prime}=0 .
$$

Substituting and resetting and separating the real and imaginary parts of equation (35) would result in 
TABLE 1: Values of parameters involved in different cases $\left(K_{1}=10\right.$ and $\left.K_{2}=100\right)$.

\begin{tabular}{ccccccccccccc}
\hline$S_{1}$ & $S_{2}$ & $\mu$ & $\eta$ & $p_{1}$ & $p_{2}$ & $c_{1}$ & $c_{2}$ & $c_{3}$ & $c_{f}$ & $\alpha_{1}$ & $\alpha_{2}$ \\
\hline \multirow{2}{*}{0.1} & \multirow{2}{*}{0} & 0.5 & 0.5 & 0.7357 & 3.9384 & 0.4568 & 2.5830 & 6.1784 & 0.0208 & 0.00338 & 2.3919 \\
& & 0.5 & 0.8 & 0.7357 & 3.9384 & 0.6521 & 6.4890 & 40.853 & 0.0208 & 0.00102 & 6.2956 \\
\hline \multirow{2}{*}{0.1} & \multirow{2}{*}{$10^{3}$} & 0.5 & 0.5 & 3.1415 & 6.1596 & 15.365 & 926.34 & 21708 & 0.4998 & 0.00007 & 23.4348 \\
& & 0.5 & 0.8 & 3.1415 & 6.1596 & 12.025 & 859.55 & 6487.6 & 0.4998 & 0.00007 & 7.5476 \\
\hline
\end{tabular}

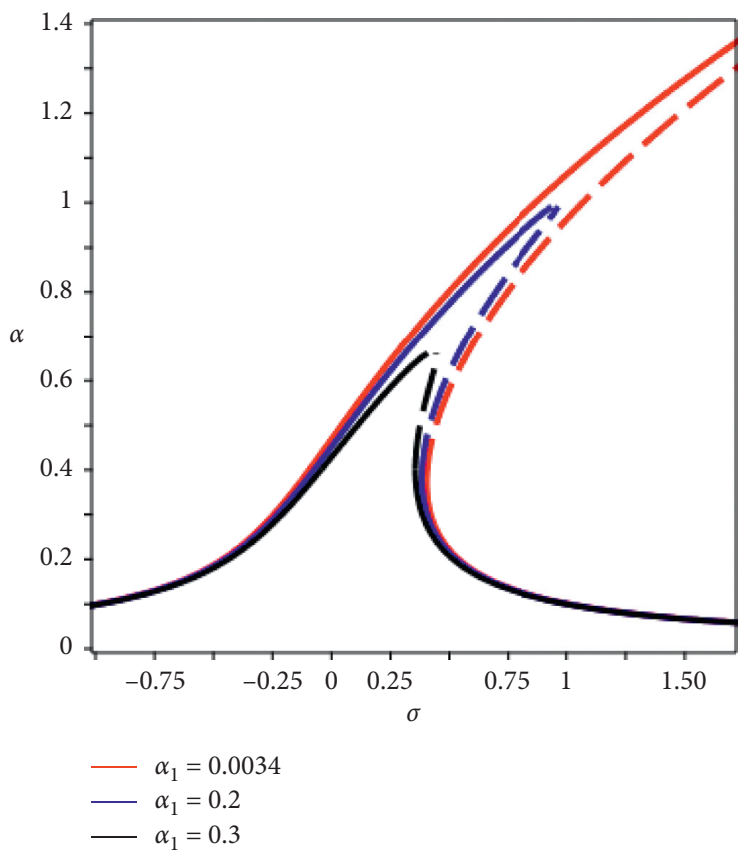

(a)

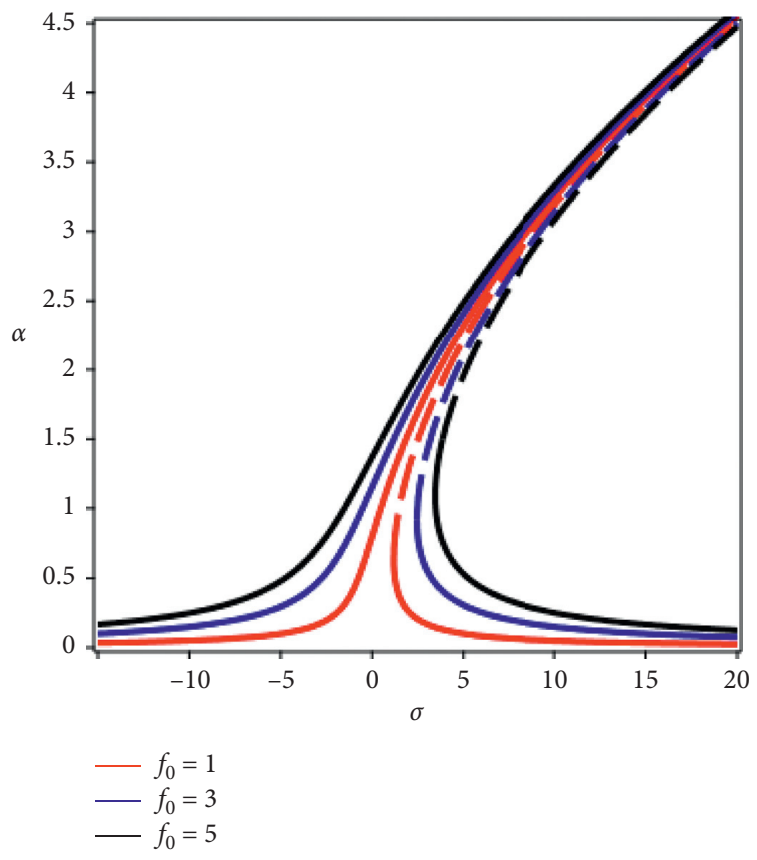

(b)

FiguRe 3: Frequency response for primary resonance. (a) $\Omega \approx 1, \alpha_{2}=2.3919$, and $f_{0}=0.2$. (b) $\Omega \approx 1, \alpha_{1}=0.0034$, and $\alpha_{2}=2.3919$. Solid and broken lines are used for the loci of stable and unstable responses, respectively.

$$
\begin{aligned}
a C^{\prime} & =-\alpha_{2} \Lambda^{3} \cos (C)-\frac{3 \alpha_{2} a^{3}}{8}+3 a \sigma-3 \alpha_{2} \Lambda^{2}, \\
a^{\prime} & =-\alpha_{2} \Lambda^{3} \sin (C)-\frac{a \alpha_{1}}{2} .
\end{aligned}
$$

\section{Results and Discussion}

In the drilling process, the stabilizer is used to keep the drillpipe in the middle of the borehole; however, a stabilizer does not provide an ability to withstand torque. From a modeling standpoint, this means that the top of the beam should be the hinged end, that is, $S_{1}=0$. At the lower end of the beam, the boundary condition depends on the type of downhole tools used there. Sometimes, the lower end can be recognized as being free (i.e., $S_{2}=0$ ) since the system's diameter is smaller than the borehole's diameter. In other situations, the contact between the drilling system and the borehole cannot be ignored. So, it is considered that $S_{2} \neq 0$. Next, two different cases are considered as follows.
4.1. Case $I\left(S_{1}=0.1\right.$ and $\left.S_{2}=0\right)$. In this condition, based on equations (9)-(11), one can determine the parameters in governing equation (7), as shown in Table 1 . The numerical results for all the resonance situations analyzed are examined here. For comparison, the parameter sensitivity study is carried out for each case. In Figure 3, the frequency response during primary resonance is shown for different values of damping $\alpha_{1}$ and forcing amplitude $f_{0}$. Due to the nonlinear support force, the amplitude-frequency curves are deflected to the right, which is related to the cubic nonlinear stiffness with hardening effect. It is clear that increasing the value of $\alpha_{1}$ and decreasing the load magnitude $f_{0}$ will both lead to the reduction of the resonance regime. Conversely, this will increase the skewness of the response curve. For a practical drilling operation, it is quite important to keep the stability of the drillstring in mind. In practical applications, one can adjust the control parameters to prevent the system from being in the unstable locations, indicated by the dashed lines in the figure.

Next, the response curves for a subharmonic resonance are considered. The response curves are quite different from what was seen before for primary resonance. In Figure 4(a), 


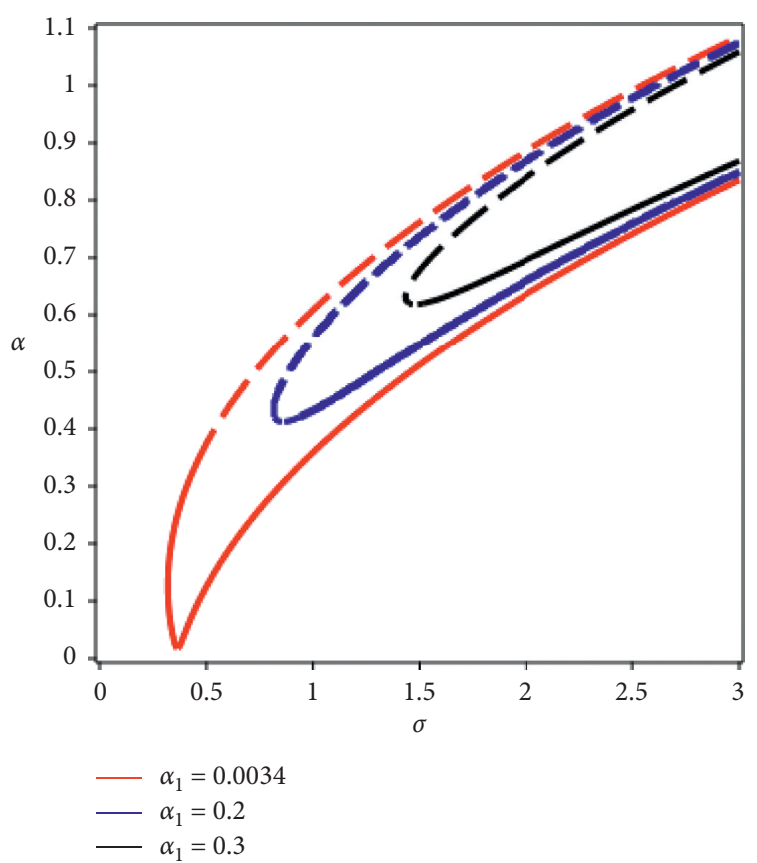

(a)

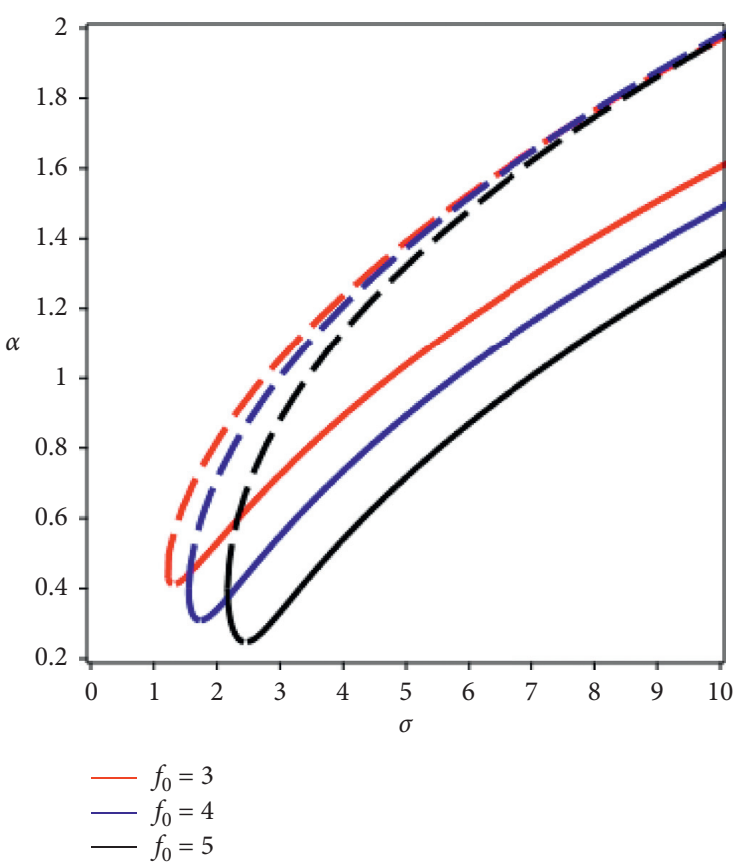

(b)

Figure 4: Frequency response for the $1 / 3$-order subharmonic resonance. (a) $\Omega \approx 3, \alpha_{2}=2.3919$, and $f_{0}=2$. (b) $\Omega \approx 3, \alpha_{1}=0.0034$, and $\alpha_{2}=2.3919$. Solid and broken lines are used for the loci of stable and unstable responses, respectively.

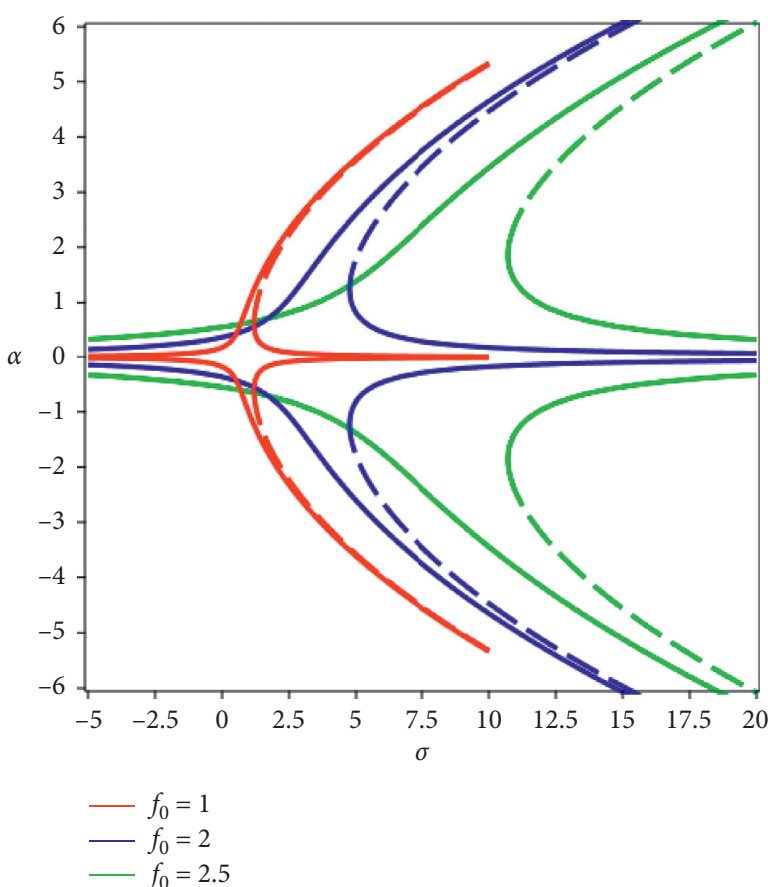

(a)

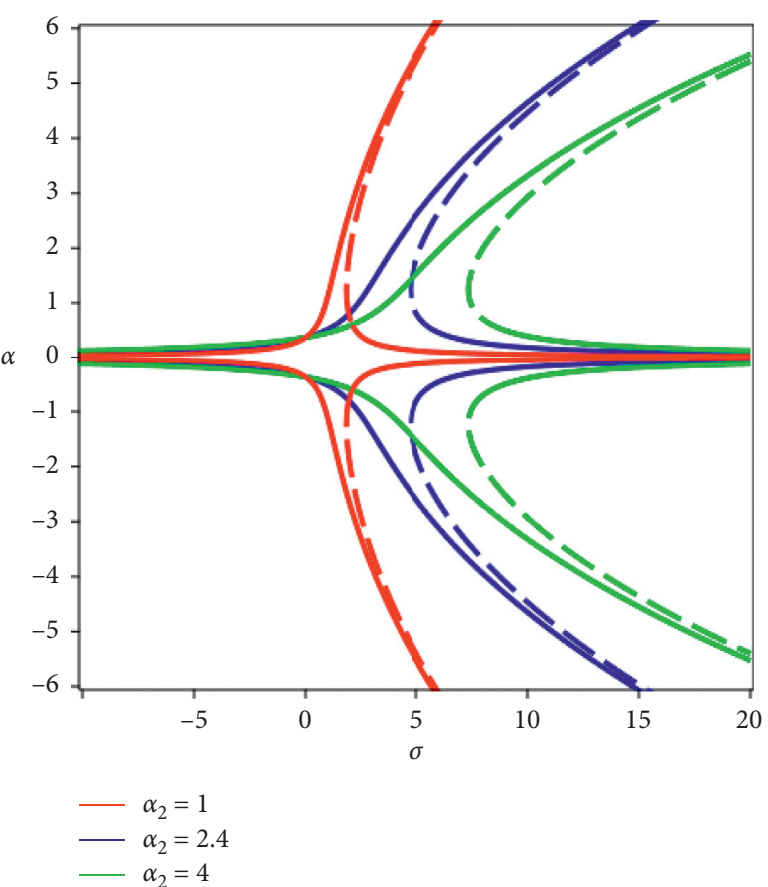

(b)

FIGURE 5: Frequency response for the third-order superharmonic resonance. (a) $\Omega \approx 1 / 3, \alpha_{1}=0.0034$, and $\alpha_{2}=2.3919$. (b) $\Omega \approx 1 / 3$, $\alpha_{1}=0.0034$, and $f_{0}=2$. Solid and broken lines are used for the loci of stable and unstable responses, respectively.

the curves have a similar structure as one changes the damping $\alpha_{1}$. As the value of $\alpha_{1}$ is increased, the resonance curve envelope gradually shrinks. In Figure 4(b), the effect of excitation load $f_{0}$ on the frequency-amplitude curve of the system is shown. As the value of $f_{0}$ is increased, the response curve not only broadens but also undergoes a lateral shift to the right. 


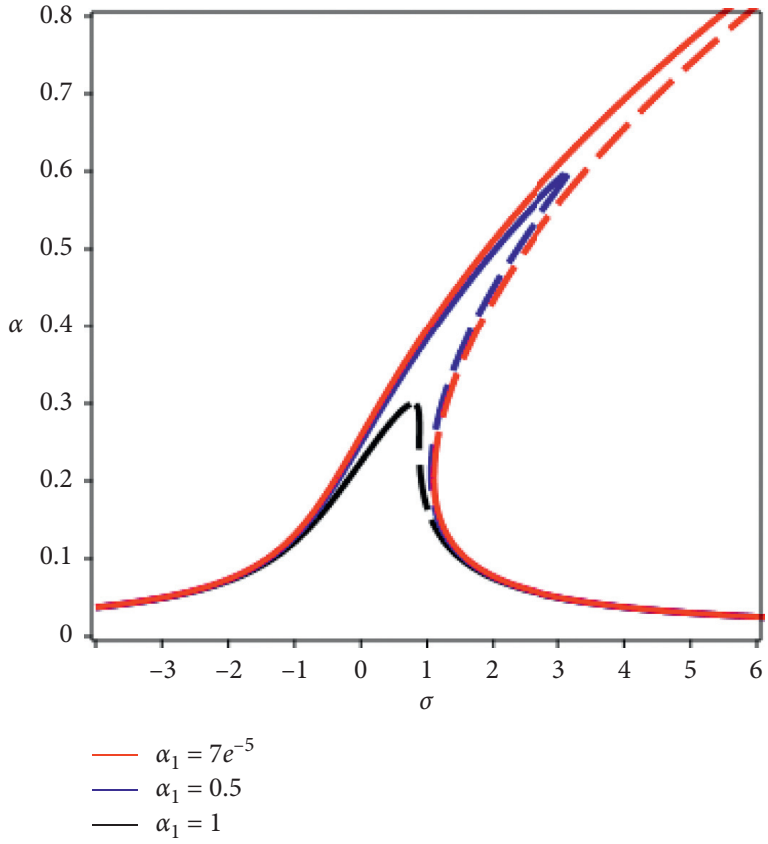

(a)

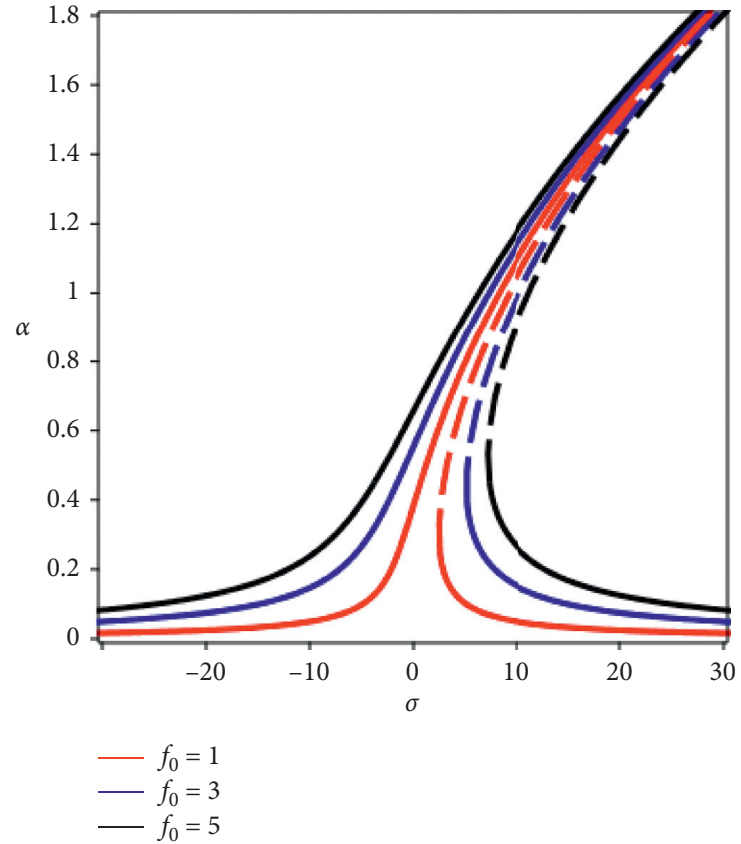

(b)

FiguRE 6: Frequency response for primary resonance. (a) $\Omega \approx 1, \alpha_{2}=23.4348$, and $f_{0}=0.3$. (b) $\Omega \approx 1, \alpha_{1}=7 e^{-5}$, and $\alpha_{2}=23.4348$. Solid and broken lines are used for the loci of stable and unstable responses, respectively.

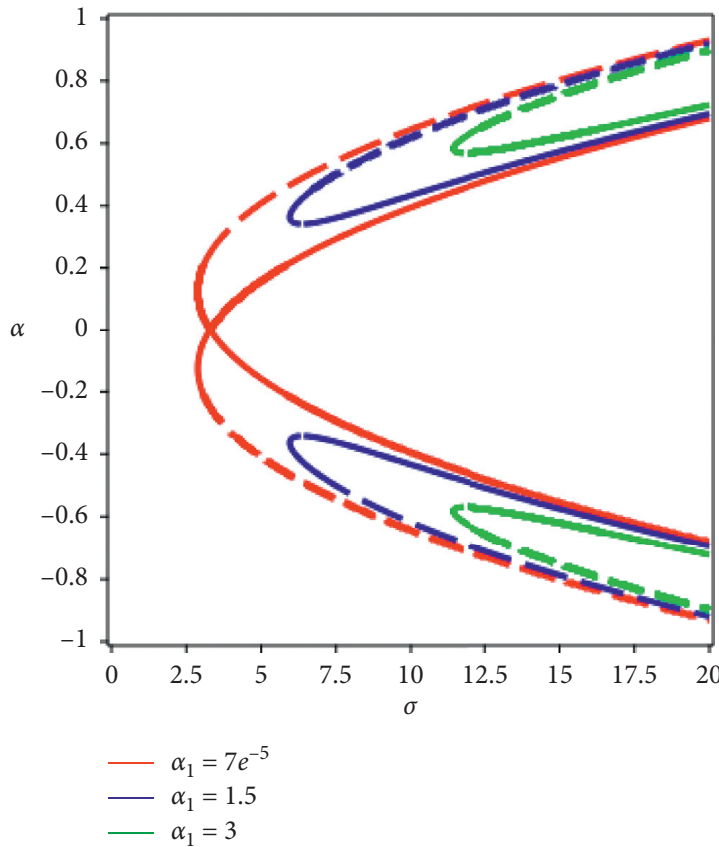

(a)

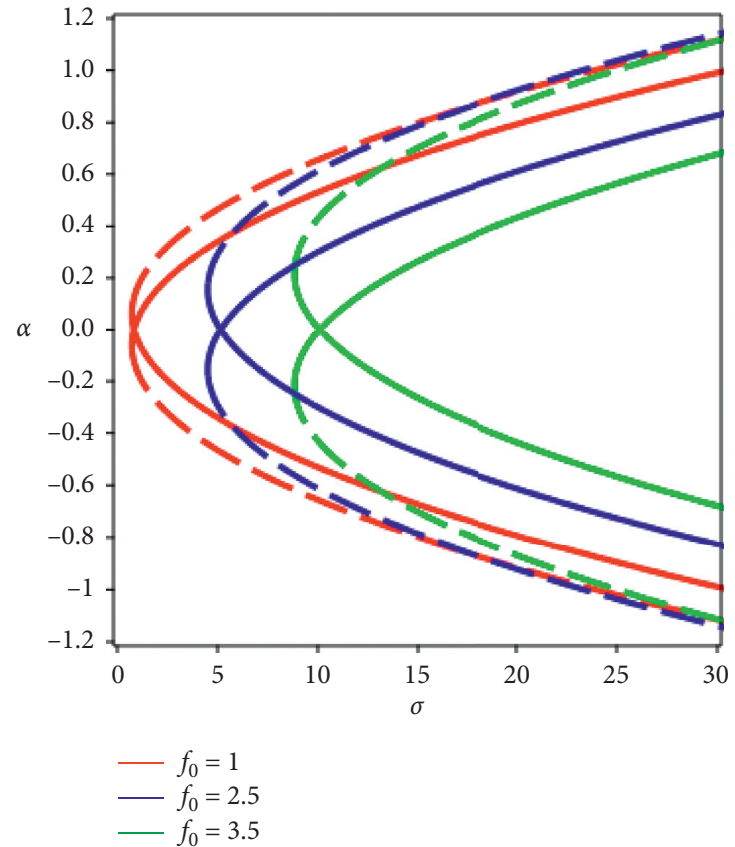

(b)

FIGURE 7: Frequency response for the $1 / 3$-order superharmonic resonance. (a) $\Omega \approx 3, \alpha_{2}=23.4348$, and $f_{0}=2$. (b) $\Omega \approx 3, \alpha_{1}=7 e^{-5}$, and $\alpha_{2}=23.4348$. Solid and broken lines are used for the loci of stable and unstable responses, respectively.

The results obtained for a superharmonic resonance case are shown in Figures 5(a) and 5(b). In this case, both damping level and forcing amplitude appear to have similar effects on the frequency response curve. As either one of them is increased, the envelope of the curve is gradually expanded, and the peaks are shifted to the right. 


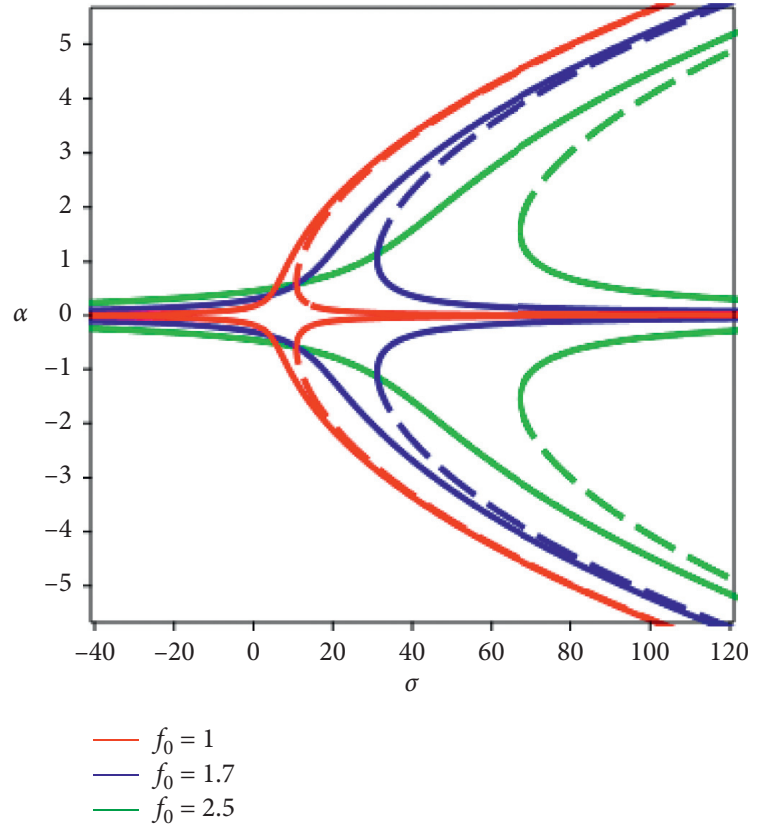

(a)

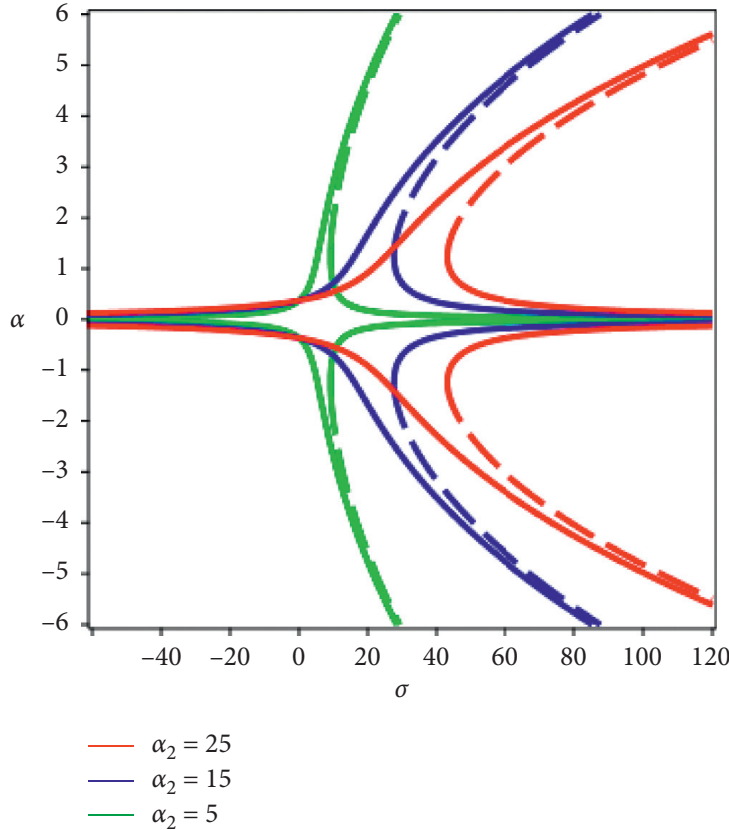

(b)

Figure 8: Frequency response for the third-order subharmonic resonance. (a) $\Omega \approx 1 / 3, \alpha_{1}=7 e^{-5}$, and $\alpha_{2}=23.4348$. (b) $\Omega \approx 1 / 3, \alpha_{1}=7 e^{-5}$, and $f_{0}=2$. Solid and broken lines are used for the loci of stable and unstable responses, respectively.

4.2. Case II $\left(S_{1}=0.1\right.$ and $\left.S_{2}=1000\right)$. In this section, the authors show the frequency responses for different resonance cases when the lower end is supported by a spring force. Similar to the first case and using the data given in Table 1, one can also employ the previous model presented in Section 3 to obtain the results of this section. In Figure 6, the authors illustrate the frequency response for primary resonance excitations. A significant difference is in the amplitude of vibration, which is lower in this case compared to that obtained in Case I. This is due to the stiffness enhancement provided by the spring support at the lower end. This finding can be useful for a designer to locate the stabilizer as well as to configure the downhole tools according to drilling conditions.

As shown in Figure 6, increasing either the value of damping parameter $\alpha_{1}$ or decreasing the excitation amplitude $f_{0}$ helps in reducing the response envelope area. For the subharmonic resonance case, as shown in Figure 7, increasing the excitation amplitude $f_{0}$ and the value of parameter $\alpha_{1}$ will cause the response curve to shift to the right. For superharmonic resonance, as shown in Figure 8, increasing the excitation amplitude $f_{0}$ and the value of parameter $\alpha_{2}$ will cause the response curve to shift to the right as well.

\section{Conclusions}

In this paper, the authors have established a reduced-order model of a drillstring section by taking the fluid loading and stabilizer effects into consideration. While the fluid loading has a direct influence on the system damping level and forcing, the presence of the stabilizer helps stiffen up the drillstring section where the stabilizer is located. After considering a single-mode approximation, the responses of the drillstring section were studied by using perturbation analyses for different resonance cases, including primary and secondary resonances. Studies have shown that, for the hinged-free case, increasing the value of $\alpha_{1}$ and decreasing the load magnitude $f_{0}$ will both lead to the reduction of the resonance regime. For the hinged-restrained case, increasing either the value of damping parameter $\alpha_{1}$ or decreasing the excitation amplitude $f_{0}$ helps in reducing the response envelope area. The findings of this study can be helpful for designers working on locating the stabilizer and configuring downhole tools. There are many avenues for future work, including consideration of multiple modes in the response.

\section{Data Availability}

All the data supporting the research in this paper are shown in the figures and tables.

\section{Conflicts of Interest}

The authors declare that they have no conflicts of interest.

\section{Acknowledgments}

The authors gratefully acknowledge the financial support from the Natural Science Foundation of China (no. 42002307), Fundamental Research Funds for the Central Universities, China (no. 2652019070), the Research Foundation of Key Laboratory of Deep Geodrilling Technology, Ministry of Natural Resources, China (no. PY201901), and 
National Key Research and Development Program of China (no. 2018YFC0603405).

\section{References}

[1] U. Ahmed and D. N. Meehan, Unconventional Oil and Gas Resources: Exploitation and Development, CRC Press, Boca Raton, FL, USA, 2016.

[2] Y. Liu and D. Gao, "A nonlinear dynamic model for characterizing downhole motions of drill-string in a deviated well," Journal of Natural Gas Science and Engineering, vol. 38, pp. 466-474, 2017.

[3] M. Sarker, D. G. Rideout, and S. D. Butt, "Dynamic model for 3D motions of a horizontal oilwell BHA with wellbore stickslip whirl interaction," Journal of Petroleum Science and Engineering, vol. 157, pp. 482-506, 2017.

[4] W. Huang and D. Gao, "Helical buckling of a thin rod with connectors constrained in a torus," International Journal of Mechanical Sciences, vol. 98, pp. 14-28, 2015.

[5] M. T. Albdiry and M. F. Almensory, "Failure analysis of drillstring in petroleum industry: a review," Engineering Failure Analysis, vol. 65, pp. 74-85, 2016.

[6] M. Jellison, J. Brock, A. Muradov, D. Morgan, and J. Rowell, "Shale play drilling challenges: case histories and lessons learned," in Proceedings of the SPE/IADC Drilling Conference, Amsterdam, The Netherlands, March 2013.

[7] L. Tan, D. Gao, and J. Zhou, "A prediction model of casing wear in extended-reach drilling with buckled drillstring," Journal of Applied Mechanics, vol. 85, no. 2, pp. 21001-21011, 2017.

[8] L. Tan and D. Gao, "Casing wear prediction model based on casing ellipticity in oil \& gas well-drilling with complex structures," Journal of Applied Mechanics, vol. 85, no. 10, pp. 101005-101016, 2018.

[9] Q. Zhang, Z. Lian, T. Lin, Z. Deng, D. Xu, and Q. Gan, "Casing wear analysis helps verify the feasibility of gas drilling in directional wells," Journal of Natural Gas Science and Engineering, vol. 35, pp. 291-298, 2016.

[10] M. C. Apostal, G. A. Haduch, and J. B. Williams, "A study to determine the effect of damping on finite-element-based, forced-frequency-response models for bottomhole assembly vibration analysis," in Proceedings of the 65th Annual Technical Conference and Exhibition. Society of Petroleum Engineers (SPE 20458), pp. 537-550, New Orleans, LA, USA, September 1990.

[11] J. D. Jansen, "Non-linear rotor dynamics as applied to oilwell drillstring vibrations," Journal of Sound and Vibration, vol. 147, no. 1, pp. 115-135, 1991.

[12] P. D. Spanos and M. L. Payne, "Advances in dynamic bottomhole assembly modeling and dynamic response determination," in Proceedings of the SPE/IADC Drilling Conference, New Orleans, LA, USA, February 1992.

[13] M. A. Vaz and M. H. Patel, "Analysis of drill strings in vertical and deviated holes using the Galerkin technique," Engineering Structures, vol. 17, no. 6, pp. 437-442, 1995.

[14] A. S. Yigit and A. P. Christoforou, "Coupled axial and transverse vibrations of oilwell drillstrings," Journal of Sound and Vibration, vol. 195, no. 4, pp. 617-627, 1996.

[15] Y. A. Khulief and H. Al-Naser, "Finite element dynamic analysis of drillstrings," Finite Elements in Analysis and Design, vol. 41, no. 13, pp. 1270-1288, 2005.

[16] S. M. Sahebkar, M. R. Ghazavi, S. E. Khadem, and M. H. Ghayesh, "Nonlinear vibration analysis of an axially moving drillstring system with time dependent axial load and axial velocity in inclined well," Mechanism and Machine Theory, vol. 46, no. 5, pp. 743-760, 2011.

[17] X. Liu, N. Vlajic, X. Long, G. Meng, and B. Balachandran, "Multiple regenerative effects in cutting process and nonlinear oscillations," International Journal of Dynamics and Control, vol. 2, no. 1, pp. 86-101, 2014.

[18] X. Liu, N. Vlajic, X. Long, G. Meng, and B. Balachandran, "Coupled axial-torsional dynamics in rotary drilling with state-dependent delay: stability and control," Nonlinear Dynamics, vol. 78, no. 3, pp. 1891-1906, 2014.

[19] M. Liao, Y. Liu, J. Páez Chávez, A. S. E. Chong, and M. Wiercigroch, "Dynamics of vibro-impact drilling with linear and nonlinear rock models," International Journal of Mechanical Sciences, vol. 146-147, pp. 200-210, 2018.

[20] S. Srivastava and C. Teodoriu, "An extensive review of laboratory scaled experimental setups for studying drill string vibrations and the way forward," Journal of Petroleum Science and Engineering, vol. 182, 2019.

[21] H. Zhang and E. Detournay, "An alternative formulation for modeling self-excited oscillations of rotary drilling systems," Journal of Sound and Vibration, vol. 474, 2020.

[22] Y. Liu, D. Gao, Z. Wei, B. Balachandran, Z. Wang, and L. Tan, "A new solution to enhance cuttings transport in mining drilling by using pulse jet mill technique," Science China Technological Sciences, vol. 62, no. 5, pp. 875-884, 2019.

[23] L. Wang and Q. Ni, "A note on the stability and chaotic motions of a restrained pipe conveying fluid," Journal of Sound and Vibration, vol. 296, no. 4-5, pp. 1079-1083, 2006.

[24] M. P. Païdoussis, Fluid-Structure Interactions: Slender Structures and Axial Flow, Elsevier, Amsterdam, Netherlands, Second edition, 2016.

[25] M. N. Hamdan and B. A. Jubran, "Free and forced vibrations of a restrained uniform beam carrying an intermediate lumped mass and a rotary inertia," Journal of Sound and Vibration, vol. 150, no. 2, pp. 203-216, 1991. 